\title{
Adaptive MIMO-of D Scheme to Reduced User Data Rate over Fading Channel
}

\author{
Rajkumar A. Veer ${ }^{1}$, Dr L C Siddanna Gowd ${ }^{2}$ \\ Research Scholar, Faculty of ECE Dept, Bharath University, Chennai, T. $\mathrm{N}^{1}$ \\ Professor, Faculty of ECE Dept, AMSEC, Namakkal, T.N²
}

\begin{abstract}
The use of multiple antennas at both the transmitter and receiver has been proposed as one of the best way to provide both an increase in reliability and also in information transmission rate. In this paper, the importance of the quantity and quality of the channel state information available at the communication ends is explored so as to achieve high data rates. The different architectures for various degrees of quality and quantity of CSI are studied in this work. The presented architectures are shown to be robust to the uncertainties of CSI. The main focus of this work is to dynamically provide resource allocation so as to achieve better QoS and improve system capacity.
\end{abstract}

Keywords: Multi-Input Multi-Output (MIMO); Orthogonal frequency division multiplexing (OFDM); Channel side information (CSI).

\section{INTRODUCTION}

An Idealized practical system assumes perfect channel state information and uses a linear transmitter to maximize the reliability of the wireless multi-antenna link. However, in actual practice the CSI is incomplete. This leads to deal with ergodic and compound capacity formulations, which arise depending on the model utilized to characterize the channel. The impact of imperfect CSI on multi-user scenario and the necessary changes required in transmission architecture so as to make it robust to the uncertainties of the side information available at both the Transmitter and receiver are studied. The logarithmic dependence of the spectral efficiency of the transmitted power makes it extremely expensive to increase the capacity by radiating more power. Also, increasing the transmitted power in a mobile terminal is not advisable due to possible violation of regulatory power masks and possible electromagnetic radiation effects. Alternately, MIMO channels exhibit a linearly increasing capacity, due to the presence of a rich scattering environment that provides independent transmission paths from each transmit to each receive antenna.

The main focus of this paper is on the characterization of the transmit covariance matrix that maximize the mutual information for the particular case of channel state uncertainty at the transmitter. Also, the power allocation strategies in a multi-user system with CSI uncertainty, so as to guarantee a certain quality of service per user are studied.

\section{ADVANTAGES OF MIMO SYSTEM}

A MIMO communication system executes an average error probability that decays as $1 /\left(\mathrm{SNR}^{\wedge} \mathrm{d}\right)$ where ' $\mathrm{d}$ ' is the diversity gain and is based on the assumption that at least one of the paths will not be in a deep fade state. Another advantage of a MIMO system is that, it is said to achieve multiplexing gain $r$, and the achievable rates scale as $[r \log$ (SNR)]. The multiplexing gain (unique for MIMO systems) is defined as the increase of the rate that can be attained through the use of multiple antennas at both sides of communication links, with respect to the rate achievable with single antenna system, without utilizing additional power.

These are shown in figure 1.

\begin{tabular}{|c|c|c|}
\hline Type & Advantage & Disadvantage \\
\hline $\begin{array}{l}\text { SISO } \\
\begin{array}{ll}\left.\left.\mathrm{Tx}^{*}\right)\right) \\
\mathrm{Rx}\end{array}\end{array}$ & $\begin{array}{l}\text { - Simplicity } \\
\text { - No } \\
\text { additional } \\
\text { processing } \\
\text { required }\end{array}$ & $\begin{array}{l}\text { - Interference } \\
\text { - Fading }\end{array}$ \\
\hline 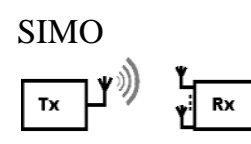 & $\begin{array}{l}\text { Easy to } \\
\text { implement }\end{array}$ & $\begin{array}{l}\text { Additional } \\
\text { processing } \\
\text { required }\end{array}$ \\
\hline 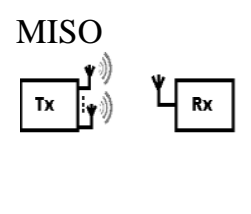 & $\begin{array}{l}\text { Processing/ } \\
\text { redundancy } \\
\text { moved from } \\
\text { receiver to } \\
\text { transmitter }\end{array}$ & \\
\hline
\end{tabular}

Figure 1 Comparison of SISO, SIMO and MISO schemes

\section{CHANNEL TYPE AND CHARACTERIZATION}

The key measure of the rates that can be achieved by any communication system on the type of channel is shown in 
IARJSET

Table 2.The receiver can either choose the best antenna to symbols(e.g., the QPSK or QAM symbols that would be receive a stronger signal or combine signals from all present in a single carrier system) at the transmitter as antennas in such a way that maximizes SNR (Signal to though they are in the frequency domain. These symbols Noise Ratio).Improves throughput and offers higher are used as inputs to the IFFT block that brings the signal diversity that leads to multiplicative increase in capacity.

Table 2 Channel type and Characterization

\begin{tabular}{|l|l|}
\hline Type of channel & Key measure of data rates \\
\hline Rapidly varying & Ergodic Capacity \\
\hline $\begin{array}{l}\text { Slow varying (or) } \\
\text { Fixed channels }\end{array}$ & Compound capacity \\
\hline
\end{tabular}

\section{MIMO-OFDM TRANSMISSION SCHEME}

The OFDM is a special type of frequency division multiplexing (FDM) wherein signals are not multiplied by a single carrier. If the FDM System had been able to use a set of subcarriers that were orthogonal to each other, a higher level of spectral efficiency could have been achieved. The guard bands that were necessary to allow individual demodulation of subcarriers in an FDM system would no longer be necessary. The use of orthogonal subcarriers would allow the subcarriers spectra to overlap, thus increasing the spectral efficiency. As long as orthogonality is maintained, it is still possible to recover the individual subcarriers signals despite their overlapping spectrums. If the dot product of two deterministic signals is equal to zero, these signals are said to be orthogonal to each other. Orthogonality can also be viewed from the standpoint of stochastic processes. If two random processes are uncorrelated, then they are orthogonal.

Each sub-set of carrier creates a sub-channel for communication.

The advantage is that

(i) They are less prone to interference, since each sub-carrier frequency is kept orthogonal to one another.

(ii) Huge bandwidth efficiency due to reduced carrier spacing (orthogonal carriers overlap)

(iii) Simple Equalization scheme or no equalization scheme

(iv) Resistant to fading

(v) Scalable data transfer rate in different channel conditions

(vi) Single Frequency Networks are possible (broadcast application)

\section{MIMO-OFDM TRANSMISSION SCHEME}

The block diagram of the MIMO-OFDM transmission scheme is shown in figure 2. In practice, OFDM systems are implemented using a combination of fast Fourier transform(FFT) and inverse fast Fourier transform(IFFT) blocks that are mathematically equivalent versions of the DFT and IDFT, respectively, but more efficient to implement. An OFDM systems treats in source into the time domain. The IFFT takes in $\mathrm{N}$ symbols at a time where $\mathrm{N}$ is the number of subcarriers in the system. Each of these $\mathrm{N}$ input symbols has a symbol period of $\mathrm{T}$ seconds. Recall that the basis functions for an IFFT are $\mathrm{N}$ orthogonal sinusoids. These sinusoids have a different frequency and the lowest frequency is DC. Each input symbol acts like a complex weight for the corresponding sinusoidal basis function. Since the input symbols are complex, the value of the symbol determines both the amplitude and phase of the sinusoid for the subcarrier. The IFFT output is the summation of all $\mathrm{N}$ sinusoids. Thus the IFFT block provides a simple way to modulate data on to $\mathrm{N}$ orthogonal subcarriers.

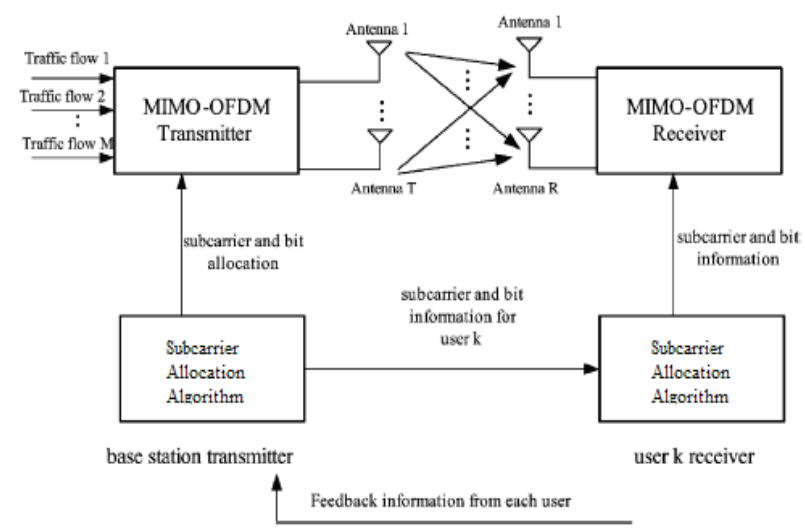

Figure 1 Bloch diagram of multiple antenna OFDM multicast system

\section{NON-IDEAL EFFECTS IN AN OFDM SYSTEM}

These effects will include impairments and receiver offsets. These effects are discussed in the following sections:

\subsection{LOCAL OSCILLATOR FREQUENCY OFFSET}

At start-up, the local oscillator (LO) frequency at the receiver is typically different from the LO frequency at the transmitter. A carrier tracking loop is used to adjust the receiver's LO frequency in order to match the transmitter LO frequency as closely as possible.

\subsection{LOCAL OSCILLATOR PHASE OFFSET}

It is also possible to have an LO phase offset, separate from an LO frequency offset. The two offsets can occur in conjunction or one or other can be present by itself. As the name suggests, an LO phase offset occurs when there is difference between the phase of the LO output and the phase of the received signal.

\subsection{FFT WINDOW LOCATION OFFSET}

Another non-ideal effect that can occur in real-world OFDM system is an effective window location offset. An 
IARJSET

In general, radio channels are fading both in time and in transmitted OFDM symbol. In practice, a correlation is often used with a known preamble sequence located at the beginning of the transmission. This correlation operation aids the receiver in synchronizing itself with the received signal's OFDM signal boundaries.

\subsection{SAMPLING FREQUENCY OFFSET}

Another potentially harmful situation is the presence of frequency. Hence, a channel estimator has to estimate tinevarying amplitudes and phases of all subcarriers. One way to do this is to use a two-dimensional channel estimator that estimates the reference values based on a few known pilot values. In this case, the signal has four subcarriers containing known pilot values to allow the estimation.To be able to interpolate the channel estimates both in time sampling frequency offset. This condition occurs when the $\mathrm{A} / \mathrm{D}$ converter output is sampled either too fast or too slow. A sampling frequency offset can be corrected by generating an error term that is used to drive a sampling rate converter.

\subsection{CARRIER INTERFERENCE}

Single-carrier interference arises from other sources that may coexist in the frequency range of interest. These can be generated by nearby circuits or other transmission sources. The single carrier system must handle this interference as a noise source for information sent. The OFDM system can avoid the frequency region of

interference by disabling or turning of the affected To determine the minimum pilot spacing in time and subcarriers. Narrow band modulated sources of frequency, we need to find the bandwidth of the channel interference can be considered similar to carrier variation in time and frequency, these bandwidths are interference in their impairment.

\section{COHERENT DETECTION}

Figure 3 shows a block diagram of a coherent OFDM receiver. After down conversion and $\mathrm{A} / \mathrm{D}$ conversion, the fast Fourier transform is used to demodulate N subcarriers of OFDM signals. For each symbol, the FFT output contains N QAM values.

However, these values contain random phase shifts and amplitude variations caused by the channel response, local oscillator drift, and timing offset. It is the task of the channel estimation block to learn the reference phases and amplitudes for all the subcarriers, such that the QAM symbols can be converted to binary soft decision.

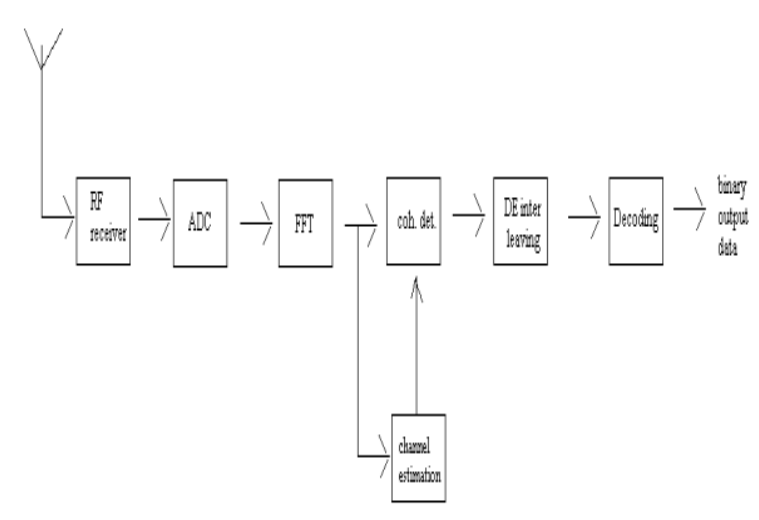

Figure 3 Block diagram of an OFDM receiver with coherent detection and frequency from the available pilots, the pilot spacing has to fulfill the Nyquist sampling theorem, which states that the sampling interval must be smaller than the inverse of the double sided bandwidth of the sampled signal. For the case of OFDM, this means that there exists both minimum subcarrier spacing and a minimum symbol spacing between pilots. By choosing the pilot spacing much smaller than these requirements, good channel estimation can be made with a relatively easy algorithm. The more pilots are used however, the smaller the effective SNR, becomes that is available for data symbols. Hence, the pilot density is a trade off between channel estimation performance and SNR loss. equal to the Doppler spread $B_{d}$ in the time domain and the maximum delay spread $\tau_{\max }$ in the frequency domain. Hence, the requirements for the pilot spacing in the time and frequency $\mathrm{s}_{\mathrm{t}}$ and $\mathrm{s}_{\mathrm{f}}$ are

$$
\begin{gathered}
\mathrm{s}_{\mathrm{t}}<1 / \mathrm{B}_{\mathrm{d}} \\
\mathrm{s}_{\mathrm{f}}<1 / \tau_{\max } .
\end{gathered}
$$

\section{RESULTS AND DISCUSSION}

\subsection{Total system data rate}

The total system data rate for the adaptive MIMO-OFDM, FDMA allocation, TDMA allocation and random user bandwidth allocation schemes are obtained and given in figure 4 to figure 7 . The average system throughput obtained for each case is given in table 2 .

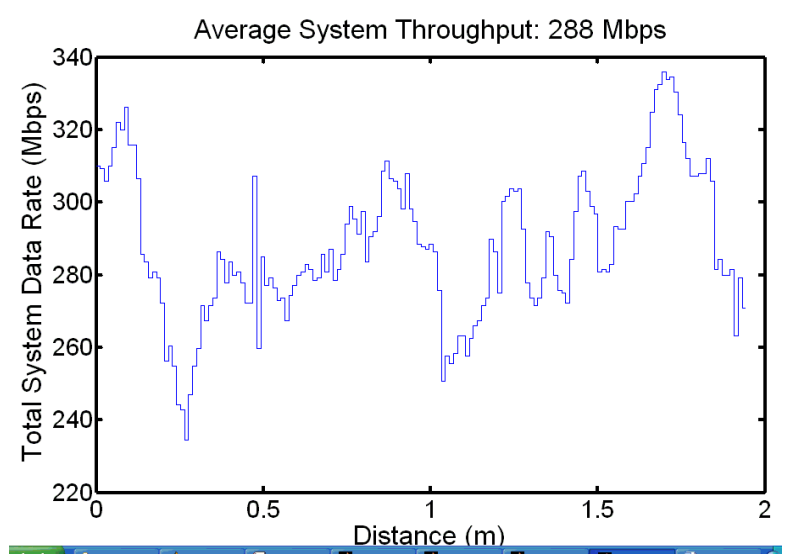

Figure 4 Average system throughput for Adaptive user bandwidth allocation MIMO-OFDM scheme 
IARJSET

National Conference on Innovative Applications and Research in Computer Science and Engineering (NCIARCSE-2017) AGTI's Dr. Daulatrao Aher College Engineering, Vidyanagar Extension, Karad

Vol. 4, Special Issue 4, January 2017

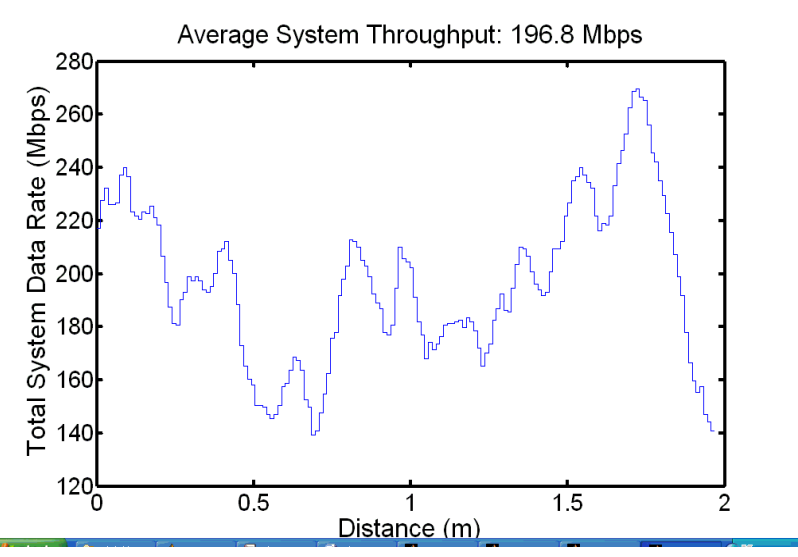

Figure 5 Average system throughput for FDMA user bandwidth allocation scheme

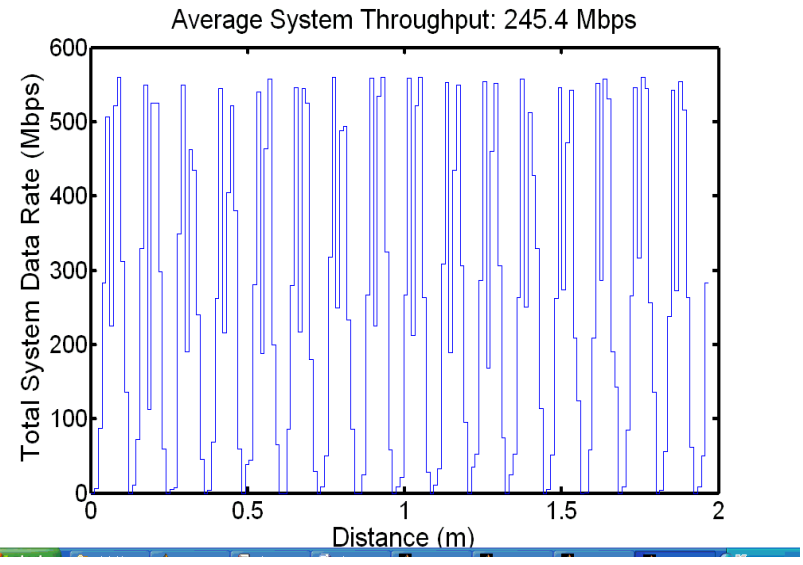

Figure 6 Average system throughput for TDMA user bandwidth allocation scheme

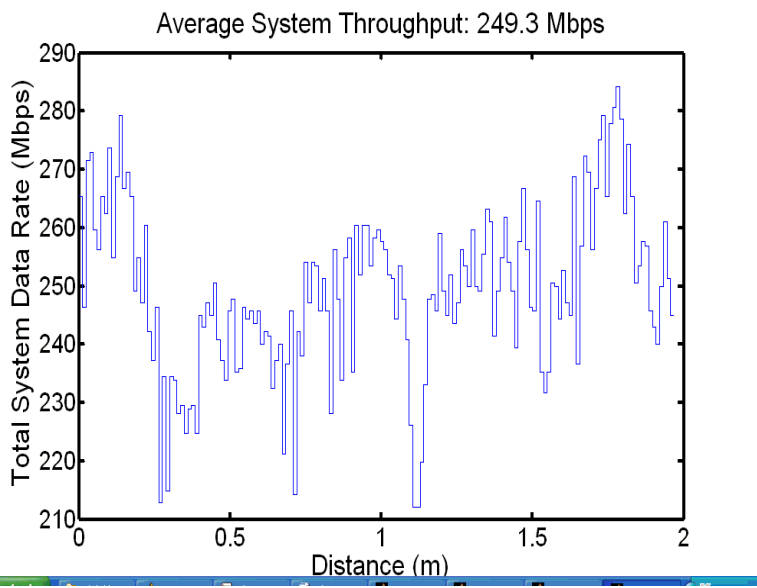

Figure 7 Average system throughput for Random user bandwidth allocation scheme

\subsection{Mean SNR variation for individual user}

The mean SNR variation for individual users for the four different schemes is presented in figure 8 to figure 11 respectively.

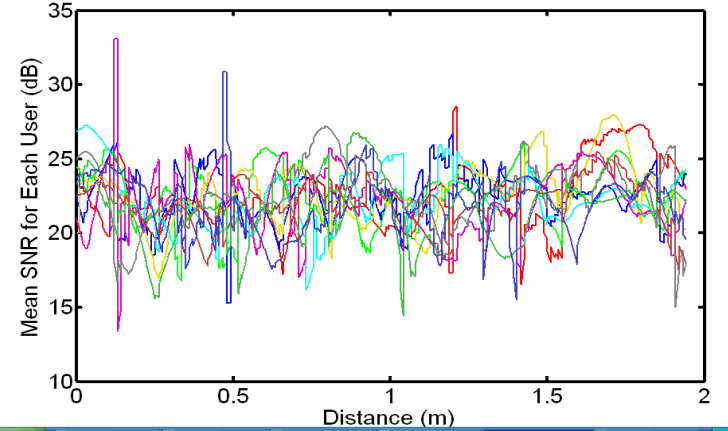

Figure 8 Mean SNR for individual user for Adaptive user bandwidth allocation MIMO-OFDM scheme

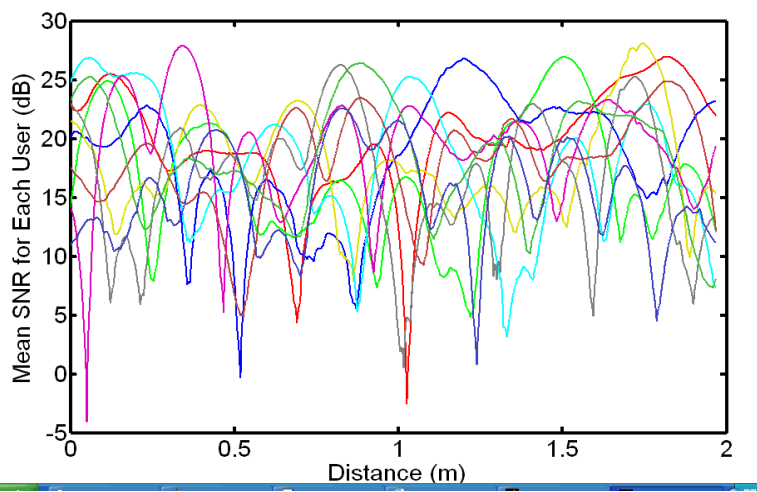

Figure 9 Mean SNR for individual user for FDMA user bandwidth allocation scheme

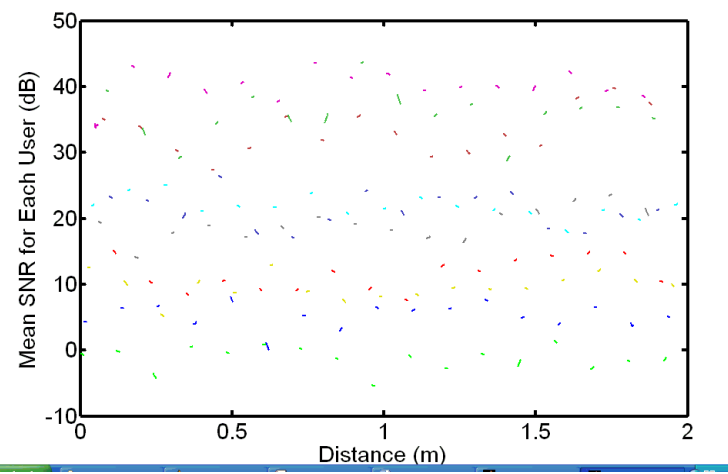

Figure 10 Mean SNR for individual user for TDMA user bandwidth allocation scheme

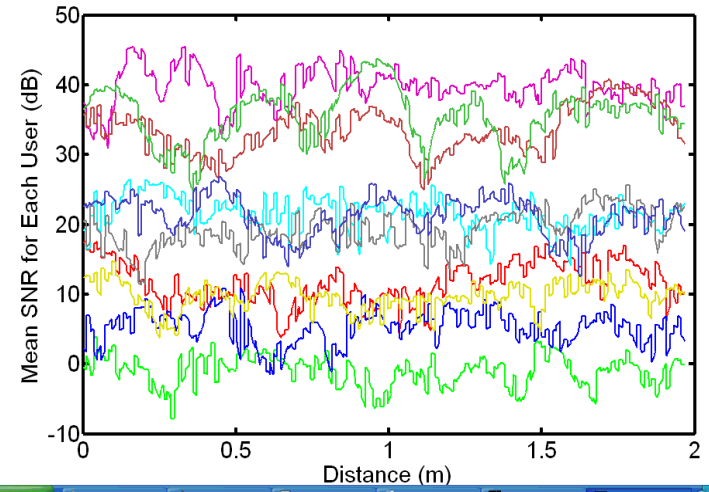

Figure 11 Mean SNR for individual user for random user bandwidth allocation scheme 
IARJSET AGTI's Dr. Daulatrao Aher College Engineering, Vidyanagar Extension, Karad

$$
\text { Vol. 4, Special Issue 4, January } 2017
$$

\subsection{USER BANDWIDTH ALLOCATIONS}

The user bandwidth allotted dynamically in the four different schemes chosen for study is presented in figure 12 to figure 15.

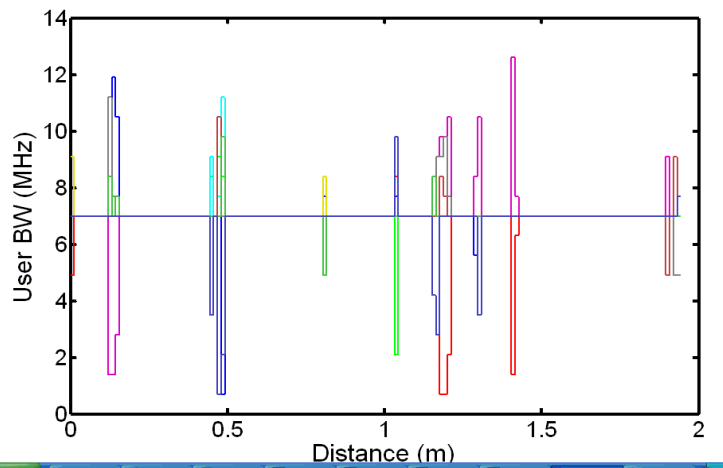

Figure 12 Adaptive User Bandwidth allocations

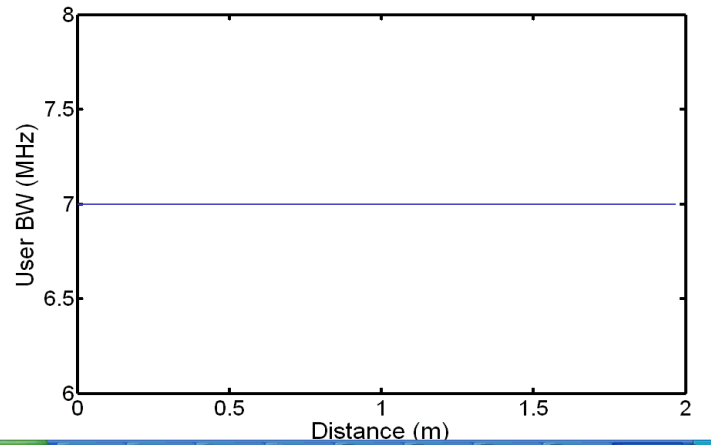

Figure 13 User Bandwidth allocations for FDMA scheme

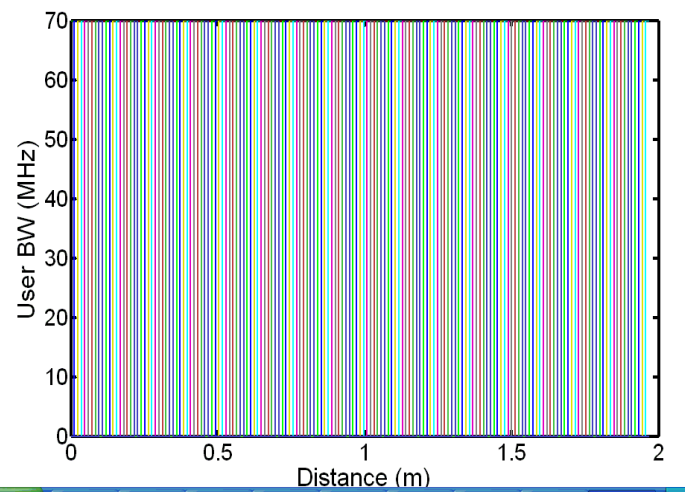

Figure 14 User Bandwidth allocations for TDMA scheme

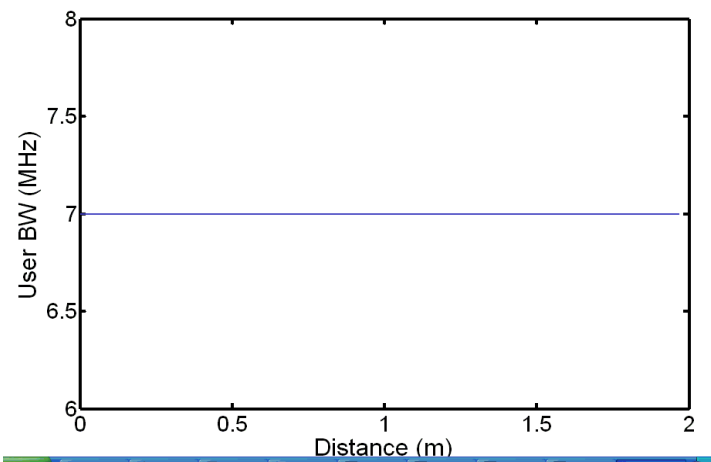

Figure 15 User Bandwidth allocations for Random scheme

\subsection{USER DATA RATE VARIATION}

The user data rate variation for the four different schemes is shown in figure 16 to figure 19.

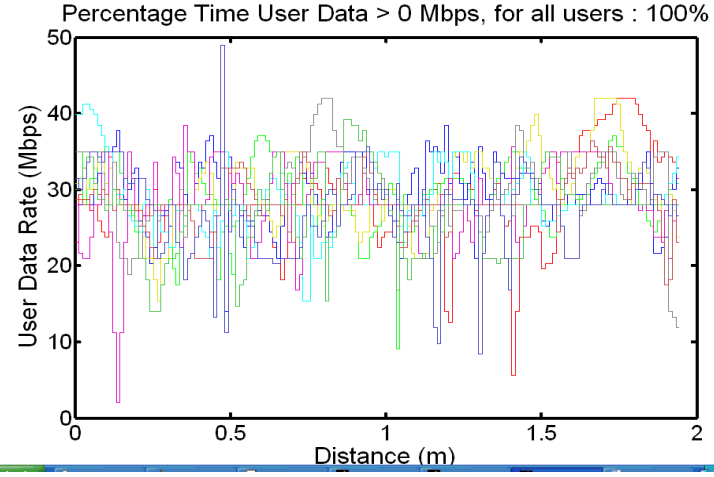

Figure 16 User data rate Variation for Adaptive MIMOOFDM scheme

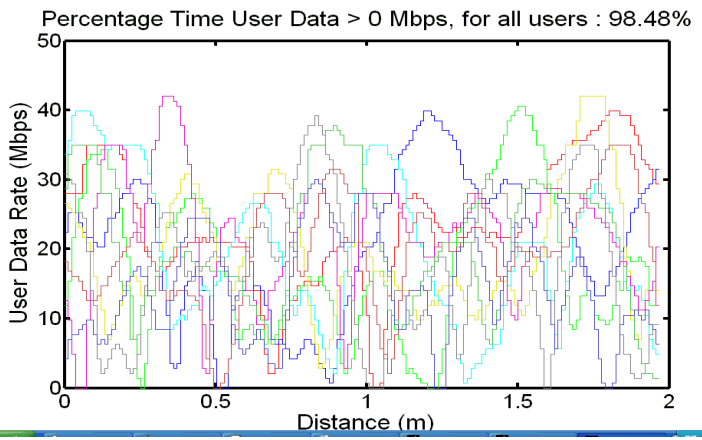

Figure 17 User data rate Variation for FDMA scheme

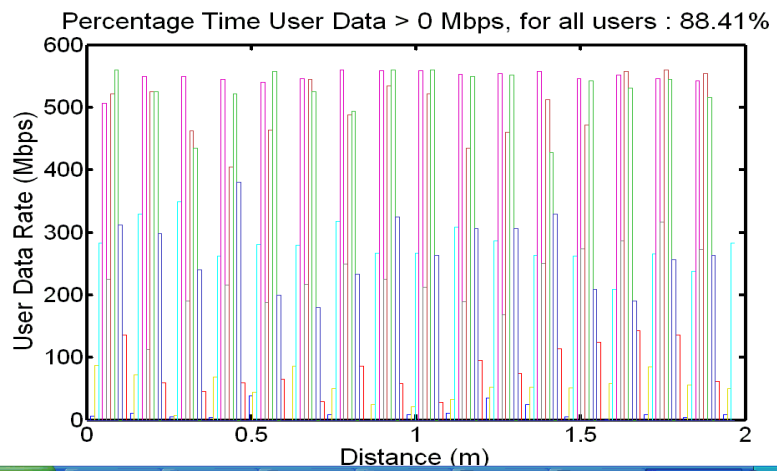

Figure 18 User data rate Variation for TDMA scheme

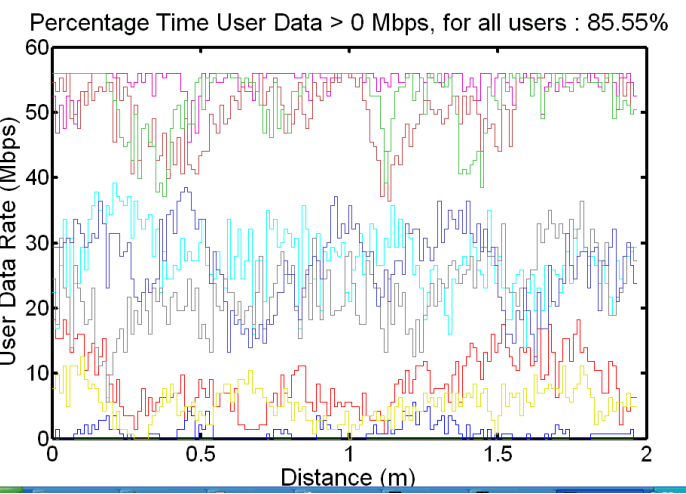

Figure 19 User data rate Variation for Random scheme 
IARJSET

\section{INFERENCE FROM RESULTS}

From the results of section 8 of this paper the performance of the four schemes are studied. It can be inferred that the adaptive user bandwidth allocation based MIMO-OFDM scheme gives the best performance compared to other schemes. It has a reduced BER, effective user bandwidth allocation, uniform user data rate variations and improved Mean SNR for individual users.

\section{CONCLUSION}

In this paper, an investigation of different adaptive techniques is presented. These techniques utilize knowledge obtained by dynamically tracking the radio channel response, to optimize the user frequency, and subcarrier modulation. Adaptive modulation independently optimizes the modulation scheme applied to each subcarrier so that the spectral efficiency is maximized, while maintaining a target Bit Error Rate (BER).

For a fading channel, adaptive modulation results in an improvement in the Signal to Noise Ratio (SNR) required to maintain a given BER, as compared with fixed modulation. Adaptive user allocation exploits the difference in frequency selective fading between users, to optimize user subcarrier allocation. In a multipath environment the fading experienced on each subcarrier varies from user to user, thus by utilizing user/subcarrier combinations that suffer the least fading, the overall performance is maximized.

\section{REFERENCES}

[1] A. Soysal and S. Ulukus. Optimum power allocation for single-user MIMO and multiuser MIMO-MAC with partial CSI. IEEE Journal on Selected Areas in Communications, 25(7):1402-1412, September 2007.

[2] M.I. Rahman, E. de Carvalho \& R. Prasad, "Impact of MIMO CoChannel Interference," in proc. 18th IEEE PIMRC'07, Athens, Greece, 3-7 September 2007.

[3] M.I. Rahman, E. de Carvalho \& R. Prasad, "Impact of MIMO CoChannel Interference," in proc. 18th IEEE PIMRC'07, Athens, Greece, 3-7 September 2007.

[4] IEEE 802.16-2005: IEEE Standard for Local and Metropolitan Area Networks - Part 16: Air Interface for Fixed and Mobile Broadband Wireless Access Systems - Amendment 2: Physical Layer and Medium Access Control Layers for Combined Fixed and Mobile Operation in Licensed Bands, February 2006.

[5] H. F. Chong, M. Motani, H. K. Garg and H. El Gamal, "On the Han- Kobayashi region for the interference channel," submitted to the IEEE Transactions on Information Theory, August 2006.

[6] M. Simon and V. Vilnrotter, "Alamouti-type space-time coding for freespace optical communication with direct detection," IEEE Trans. On Wireless Communications, vol. 4, no. 1, pp. 35-39, 2005.

[7] A. Goldsmith, Wireless Communications, Cambridge University Press, 2005.

[8] M. Sharif and B. Hassibi, "On the Capacity of MIMO broadcast channels with partial side information," IEEE Trans. Infom. Theory, vol. 51, no. 2, pp.506-522, Feb. 2005.

[9] C. Yuen, Y. L. Guan and T. T. Tjhung, "Quasi-orthogonal STBC with minimum decoding complexity," IEEE Trans. Wireless Commun., vol. 4, no.5, pp. 2089-2094, Sep. 2005.
[10] J. Z. Ying and K. B. Letaief, "An efficient resource-allocation scheme for spatial multi-user access in MIMO/OFDM systems," IEEE Trans. Comm., vol. 53, no.1, pp. 107- 116, Jan. 2005.

[11] M. L. McCloud, "Analysis and design of short block OFDM spreading matrices for use on multipath fading channels," IEEE Trans. Commun., vol. 53, no. 4, pp. 656-665, Apr. 2005.

[12] J. Z. Ying and K. B. Letaief, "Adaptive resource allocation for multi-access MIMO/OFDM systems with matched filtering, " IEEE Trans. Comm., vol. 53, no. 11, pp. 1810- 1816, Nov. 2005.

[13] Zentner. R, Nagy R, Zentner E: Elliptical Single- bounce Model for MIMO Channel Simulations", COST273, Germany, 2004.

[14] Y. Alqudah, M. Kavehrad, and S. Jivkova, "Optical wireless multispot diffusing; a mimo configuration," IEEE International Conference on Communications, vol. 6, pp. 3348 - 3352, June 2004.

[15] S. Zhou, P. Xia, G. Leus, and G. B. Giannakis, "Chip-interleaved block spread CDMA versus DS-CDMA for cellular downlink: a comparative study," IEEE Trans. Wireless Commun., vol. 3, no. 1, pp. 176-190, Jan. 2004

[16] Cho, M., Cho, H., Ro, S., and Hong, D.: 'A novel time spreading method for down-link OFDM-coded division multiplexing systems'. Proc. IEEE VTC, April 2003, pp. 764-768.

[17] A.J. Paulraj, R. Nabar \& D. Gore, Introduction to Space-Time Wireless Communications, 1st ed. Cambridge University Press, September 2003.

[18] L. Zheng, M. M'edard, D. Tse, and C. Luo, "On Channel Coherence in the Low SNR Regime", in Proc. Allerton Conf., 2003.

[19] A.Sendonaris, E. Erkip, and B. Aazhang, "User cooperation diversity- 20 Part I: System description," IEEE Trans. Commun., vol. 51, no. 11, pp. 1927-1938, Nov. 2003.

[20] "User cooperation diversity Part II: Implementation aspects and performance analysis, IEEE Trans. Commun., vol. 51, no. 1, pp. 1939-15' '1948, Nov. 2003.

[21] A. Bury, J. Egle, and J. Lindner, "Diversity comparison of spreading transforms for multicarrier spread spectrum transmission," IEEE Trans.Commun., vol. 51, no. 5, pp. 774-781, May 2003.

[22] T. E. Hunter and A. Nosratinia, "Cooperation diversity through coding," in IEEE Int. Symp. Inform. Theory, 2002, p. 220.

[23] J. Kim and J. M. Cioffi, "Spatial Multi-user Access with Antenna Diversity using Singular Value Decomposition," Proc. IEEE Int'l. Conf Communications, '00, pp. 1253-1257, Jun. 2000.

[24] J. Garcia-Luna-Aceves and J. Raju, "Distributed assignment of codes for multihop packet-radio networks," in Proc. IEEE MILCOM, Monterey, California, Nov 1997.

[25] A. A. Bertossi and M. A. Bonuccelli, "Code assignment for hidden terminal interference avoidance in multihop packet radio networks," IEEE/ACM Transactions on Networking, vol. 3, no. 4, pp. 441-449, Aug 1995.

[26] L. Hu, "Distributed code assignments for CDMA packet radio networks," IEEE/ACM Transactions on Networking, vol. 1, no. 6, pp. 668-677, Dec 1993.

[27] Yang Han, See Ho Ting and Yong Liang Guan. A High Rate OpenLoop MIMO Multi-User Downlink Transmission System. 